\title{
Analysis of quality of life after randomized controlled trial of alpha-l adrenoceptor antagonist alone and in combination with cyclooxygenase-2 inhibitor in patients who underwent low-dose-rate brachytherapy for prostate cancer
}

\author{
Yasushi Nakai, MD, PhD', Nobumichi Tanaka, MD, PhD', Isao Asakawa, MD, PhD², Kazumasa Torimoto, MD, PhD', \\ Makito Miyake, MD, PhD', Satoshi Anai, MD, PhD', Tomomi Fujii, MD, PhD³, Masatoshi Hasegawa, MD, PhD², \\ Kiyohide Fujimoto, MD, PhD' \\ 'Department of Urology, Nara Medical University, Nara, Japan, ${ }^{2}$ Department of Radiation Oncology, Nara Medical University, Nara, Japan, \\ ${ }^{3}$ Department of Pathology, Nara Medical University, Nara, Japan
}

\begin{abstract}
Purpose: The goal of this study was to evaluate the effect of cyclooxygenase-2 (COX-2) inhibitors on quality of life (QoL) of patients undergoing low-dose-rate (LDR) brachytherapy.

Material and methods: A total of 310 patients with prostate cancer who had undergone LDR brachytherapy were enrolled. The patients were randomized $(1: 1)$ to the monotherapy group (tamsulosin alone: $0.2 \mathrm{mg} / \mathrm{day}, n=156$ ) and the combination group (tamsulosin: $0.2 \mathrm{mg} /$ day plus celecoxib: $200 \mathrm{mg} / \mathrm{day}, n=154$ ) without placebo. Using the expanded prostate cancer index composite (EPIC) and medical outcomes study 8-item short form health survey (SF-8) questionnaire, QoL was evaluated at baseline and at 1,3, 6, and 12 months after seed implantation.

Results: The mean changes in scores from baseline to 1 and 3 months after seed implantation for the urinary (1M: $-10.5,3 \mathrm{M}:-10.9)$ and bowel (1M: -2.4, 3M: -4.2) domains of EPIC in the combination group were not significantly different from those (urinary 1M: -11.0 , 3M: -11.4 , bowel 1M: $-2.3,3 \mathrm{M}:-4.6$ ) in the monotherapy group. The mean changes in scores from baseline to 1 and 3 months after seed implantation for the physical component summary (1M: 0.009, 3M: -0.32) and mental component summary (1M: 0.41, 3M: 0.36) of SF-8 in the combination group were not significantly different from those (physical component $1 \mathrm{M}:-0.89,3 \mathrm{M}:-0.22$, mental component $1 \mathrm{M}: 1.3,3 \mathrm{M}: 1.1$ ) in the monotherapy group.

Conclusions: Combination treatment with celecoxib and tamsulosin during the peri-operative period is not warranted for improving QoL in patients undergoing LDR brachytherapy.

J Contemp Brachytherapy 2019; 11, 5: 409-416 DOI: https://doi.org/10.5114/jcb.2019.88142
\end{abstract}

Key words: cyclooxygenase-2 inhibitor, low-dose-rate brachytherapy, prostate cancer, quality of life.

\section{Purpose}

Low-dose-rate (LDR) brachytherapy for prostate cancer has good oncological control, similar to radical prostatectomy and intensity-modulated radiation therapy (IMRT) in patients with low- and intermediate-risk $[1,2,3]$. Furthermore, a combination of external beam radiation therapy (EBRT) and LDR brachytherapy appears to have outcomes superior to those of surgery alone or EBRT alone in high-risk patients $[1,2,3,4]$. However, patients undergoing LDR brachytherapy have a lower QoL with respect to urinary, bowel, and sexual functions for
6 months after LDR brachytherapy [5,6]. Alpha-1 adrenoceptor antagonist administration significantly improves urinary symptoms in patients treated with LDR brachytherapy $[7,8]$. However, a low QoL is not sufficiently improved in patients taking alpha-1 adrenoceptor antagonist alone after LDR brachytherapy [9]. Therefore, we conducted a randomized controlled trial comparing alpha-1 adrenoceptor antagonist administration alone and in combination with cyclooxygenase-2 (COX-2) inhibitor in patients who had undergone LDR brachytherapy for prostate cancer, with a primary endpoint of change in the international prostate symptom score (IPSS)
Address for correspondence: Nobumichi Tanaka, Department of Urology, Nara Medical University, 840 Shijo-cho, Nara, 634-8522, Japan, phone: +81-744-22-3051 (ext. 2338), fax: +81-744-22-9282,

e-mail: sendo@naramed-u.ac.jp
Received: 10.01 .2019

Accepted: 19.08 .2019

Published: 30.10 .2019 
at 3 months after LDR brachytherapy [10]. In patients treated with COX-2 inhibitor, no change was observed in the IPSS, but daytime urinary frequency and post-void residual after seed implantation reduced.

If inflammation caused by LDR brachytherapy (needle insertion and seed implantation) or radiation lowers quality of life, anti-inflammatory agents could improve QoL. An in vivo study revealed that irradiation for bladder carcinoma causes pronounced COX-2 dependent inflammatory changes in the bladder wall, and that COX-2 inhibitors can decrease adverse events caused by radiation [11]. Feigenberg et al. retrospectively evaluated the efficacy of COX-2 inhibitors in patients with prostate cancer treated with brachytherapy, and found that treatment with COX-2 before brachytherapy significantly reduces urinary retention [12]. Furthermore, COX-2 inhibitor use improved the lowered QoL caused by radiation therapy in patients with nasopharyngeal carcinoma and breast cancer $[13,14]$. However, there is no study that evaluates the efficacy of COX-2 inhibitor in terms of QoL after LDR brachytherapy. Therefore, the present study aimed to focus on the additional impact of COX-2 inhibitor on QoL of patients undergoing LDR brachytherapy using the expanded prostate cancer index composite (EPIC) [15] and medical outcomes study 8-item short form health survey (SF-8) questionnaires [16].

\section{Material and methods}

\section{Eligibility, registration, stratification, and randomization}

Complete information on eligibility, registration, stratification, randomization, and the a priori statistical model has been provided in a previous publication discussing the change in IPSS [10]. The sample size was calculated ( $\alpha$ : $0.05, \beta: 0.8$, the mean difference of IPSS at 3 months after LDR brachytherapy in 2 groups: 2.5, standard deviation: 8.0) [6,17] and 162 patients were required for each group. A total of 360 patients with localized prostate cancer who had undergone LDR brachytherapy between May 2010 to July 2013 were enrolled in this open-labeled randomized controlled trial. Written informed consent was provided by 310 patients who were allocated to treatment with either tamsulosin alone $(0.2 \mathrm{mg} /$ day), or tamsulosin $(0.2 \mathrm{mg} /$ day $)$ and celecoxib (200 mg/day) without placebo. This trial is registered with the UMIN Clinical Trials Registry as UMIN000003649. Treatment with tamsulosin began a day after brachytherapy started, and continued for at least 6 months after seed implantation or until the IPSS returned to the pretreatment score or lower, whereas celecoxib treatment was activated a day after brachytherapy started and continued for 3 months. The EPIC (disease-related QoL) and short form-8 (SF-8 health-related QoL) questionnaires were administered before seed implantation and at 1, 3, 6, and 12 months after seed implantation. The urologist who assessed the results of the questionnaires was not the same as the one who initiated the medication.

\section{Radiation therapy}

The prescribed dose of ${ }^{125} \mathrm{I}$ seed implantation alone was 160 Gy $[18,19]$, whereas that in combination with EBRT was 110 Gy $[18,19]$. The clinical target volume for the LDR brachytherapy included the whole prostate. The implantation was based on peripheral loading technique with real time dynamic dose calculation using VariSeed ${ }^{\circledR}$ 8.0 (Varian Medical Systems, Palo Alto CA, USA). The target for the EBRT was determined 1 month after seed implantation, and the patients received 45 Gy ( 25 fractions, 1.8 Gy per fraction) using 10-MV photon energy and the three-dimensional conformal technique. The clinical target volume included both the entire prostate and the proximal third of the seminal vesicles. Post-implant dosimetry analysis was performed at 1-month post-seed implant.

\section{Statistical analysis}

Statistical analyses were carried out with PRISM software, version 7.00 (San Diego, CA, USA). A previous trial was powered according to the primary endpoint of change in the IPSS at 3 months after seed implantation. Secondary endpoints were QoL scores. Baseline patient and treatment characteristics were compared using the Chi-square test for categorical variables and the unpaired $t$-test was used for continuous variables. Changes in the QoL scores were calculated by subtracting the baseline score from the score at a designated time period for each patient. The mean change in scores was calculated for each time period. Statistically significant differences in the mean changes in scores for the urinary, bowel, hormonal, and sexual functions from the EPIC, physical component summary (PCS), and mental component summary (MCS) of the SF-8 questionnaires between the two treatment groups were assessed using an unpaired $t$-test.

\section{Results}

Patients who had provided written consent for the present trial were randomized to either the combination group $(n=154)$ or to the monotherapy group $(n=156)$. No adverse events related to COX-2 inhibitor use was observed, and no patient discontinued taking celecoxib due to adverse events. Moreover, no patient from the monotherapy group was administered celecoxib because of urinary disorders. There were no significant differences between the two groups with regard to patient background (Table 1) or post-implant dosimetry parameters (Table 2). The completeness of the EPIC and SF-8 data was excellent at $99.5 \%$ (1,543/1,550 points). There were no items with $5 \%$ or more values missing. There were no significant differences between the two groups in any domain, except for the physical domain score $(p=0.01)$ (Table 3).

For the urinary domain of EPIC, the mean change in scores from baseline to 1 month in the combination and monotherapy groups were -10.5 (standard deviation [SD]: \pm 11.7 ) and -11.0 (SD: \pm 15.3 ), respectively, and no significant difference was found between the two groups $(p=0.75)$. The mean change in scores from baseline to 3 months in the combination and monotherapy groups 
Table 1. Patients characteristics

\begin{tabular}{lccc} 
Variables & $\begin{array}{c}\text { Combination group } \\
(n=154)\end{array}$ & $\begin{array}{c}\text { Monotherapy group } \\
(n=156)\end{array}$ & $P$ \\
\cline { 2 - 4 } & \multicolumn{2}{c}{ Median (range) or $n$} & 0.91 \\
\hline Age (yrs) & $70(48-80)$ & $70(52-81)$ & 0.68 \\
\hline PSA (ng/ml) & $7.0(3.7-43.6)$ & $6.6(1.2-41.7)$ & 0.18 \\
\hline Prostate volume $(\mathrm{ml})$ & $22(9-43)$ & $22(9-49)$ & 0.54 \\
\hline Stage T1c/T2a/T2b/T2c/T3a & $75 / 52 / 12 / 9 / 6$ & $79 / 52 / 17 / 5 / 3$ & 0.96 \\
\hline Gleason score 6/7/8-10 & $60 / 87 / 7$ & $62 / 87 / 7$ & 0.57 \\
\hline Neo ADT, yes/no & $69 / 85$ & $64 / 92$ & 0.07 \\
\hline Duration of neo ADT (months) & $4(2-72)$ & $4(1-17)$ & 1.00
\end{tabular}

Neo ADT - neoadjuvant androgen deprivation therapy, PSA - prostate-specific antigen, EBRT - external beam radiation therapy

Table 2. Post-implant dosimetry parameters

\begin{tabular}{lccc} 
Variables & $\begin{array}{c}\text { Combination group } \\
(n=154)\end{array}$ & $\begin{array}{c}\text { Monotherapy group } \\
(n=156)\end{array}$ & $P$ \\
\cline { 2 - 4 } & \multicolumn{2}{c}{ Median (range) } & 0.44 \\
\hline $\mathrm{D}_{90}(\%)$ & $122.5(105-138)$ & $121.6(104-138)$ & 0.82 \\
\hline $\mathrm{D}_{90}(\mathrm{~Gy})$ & $183.4(107-221)$ & $182(124-217)$ & 0.69 \\
\hline $\mathrm{V}_{150}(\%)$ & $99.2(96-100)$ & $99.1(95-100)$ & 0.54 \\
\hline$\% \mathrm{DD}_{5}(\%)$ & $57.4(32-78)$ & $56.1(34-74)$ & 0.67 \\
\hline $\mathrm{UD}_{5}(\mathrm{~Gy})$ & $137.4(110-188)$ & $136.2(111-179)$ & 0.73 \\
\hline$\% \cup D_{30}(\%)$ & $199.4(126-300)$ & $198.7(127-277)$ & 0.69 \\
\hline $\mathrm{UD}_{30}(\mathrm{~Gy})$ & $125.5(104-150)$ & $125.1(105-143)$ & 0.86 \\
\hline$\% \mathrm{DD}_{90}(\%)$ & $187.2(118-241)$ & $184.7(124-229)$ & 0.71 \\
\hline $\mathrm{UD}_{90}(\mathrm{~Gy})$ & $77.8(26.1-123)$ & $77.8(24.5-119)$ & 0.55 \\
\hline $\mathrm{R}_{100}(\mathrm{ml})$ & $109.5(31-184)$ & $111.0(28-1091)$ & 0.99
\end{tabular}

$\% D_{90}, D_{90}$ - minimal percentage of the dose and minimal dose (Gy) received by $90 \%$ of the prostate, $V_{100}, V_{150}$ - percentage of the prostate volume receiving $100 \%$ and $150 \%$ of the prescribed minimal peripheral dose, $\% \cup D_{5}, U D_{5}-$ minimal percentage of the dose and minimal dose (Gy) received by $5 \%$ of the urethra, $\% \cup D_{30}$, $U D_{30}$ - minimal percentage of the dose and minimal dose (Gy) received by $30 \%$ of the urethra, $\% \cup D_{90}, U D_{90}$ - minimal percentage of the dose and minimal dose (Gy) received by $90 \%$ of the urethra, $R_{100}$ - rectal volume $(\mathrm{ml})$ receiving $100 \%$ of the prescribed dose

were -10.9 (SD: \pm 12.0$)$ and -11.4 (SD: \pm 15.4$)$, respectively, without a significant difference $(p=0.75)$. There was no significant difference between the mean change in scores from baseline to 6 months $(p=0.39$, combination: mean $-7.7, \mathrm{SD}: \pm 11.4$; monotherapy: mean $-7.8, \mathrm{SD}: \pm 16.2$ ) or 12 months $(p=0.64$, combination: mean -5.3, SD: \pm 15.1 ; monotherapy: mean $-3.7, \mathrm{SD}: \pm 14.9$ ). The chronological changes in the subscales of the urinary domain are shown in Figure 1.

For the bowel domain of EPIC, the mean change in scores from baseline to 1 month in the combination and monotherapy groups were -2.4 (SD: \pm 7.9 ) and -2.3 (SD: \pm 8.2$)$, respectively, and no significant difference was found between the two groups $(p=0.95)$. The mean change in scores from baseline to 3 months in the combination and monotherapy groups were $-4.2(\mathrm{SD}: \pm 9.4)$ and -4.6 (SD: \pm 9.4 ), respectively, without a significant difference $(p=0.88)$. There was no significant difference between the mean changes in scores from baseline to 6 months $(p=0.77$, combination: mean -3.5 , SD: \pm 10.3 ; monotherapy: mean -3.9 , SD: \pm 11.3 ) or 12 months ( $p=0.28$, combination: mean -3.7 , SD: \pm 14.5 ; monotherapy: mean -2.2 , SD: \pm 9.9 ). The chronological changes in the subscales of the bowel domain are shown in Figure 2.

For the hormone domain of EPIC, the mean changes in scores from baseline to 1 month in the combination and monotherapy groups were 0.80 (SD: \pm 7.9 ) and 1.42 (SD: \pm 7.9$)$, respectively, and no significant difference was found between the two groups $(p=0.49)$. The mean change in scores from baseline to 3 months in the combination 
Table 3. EPIC and SF-8 scores at baseline

\begin{tabular}{lccc} 
Variables & $\begin{array}{c}\text { Combination group } \\
(n=154)\end{array}$ & $\begin{array}{c}\text { Monotherapy group } \\
(n=156)\end{array}$ & $P$ \\
\cline { 2 - 3 } & & & \\
\hline EPIC & & & \\
\hline Urinary domain & $96.0( \pm 5.2)$ & $96.2( \pm 5.3)$ & 0.74 \\
\hline Bowel domain & $95.4( \pm 5.2)$ & $95.6( \pm 5.8)$ & 0.75 \\
\hline Hormone domain & $93.0( \pm 7.5)$ & $93.1( \pm 8.1)$ & 0.91 \\
\hline Sexual domain & $40.6( \pm 15.0)$ & $39.0( \pm 12.5)$ & 0.31 \\
\hline SF-8 & & 0.38 \\
\hline Physical function & $48.6( \pm 6.9)$ & $49.3( \pm 7.0)$ & 0.01 \\
\hline Role physical & $48.5( \pm 7.3)$ & $50.4( \pm 5.9)$ & 0.20 \\
\hline Bodily pain & $53.0( \pm 7.5)$ & $54.1( \pm 7.7)$ & 0.40 \\
\hline Vitality & $51.2( \pm 6.1)$ & $51.8( \pm 6.5)$ & 0.24 \\
\hline General health & $50.0( \pm 6.0)$ & $50.8( \pm 6.0)$ & 0.48 \\
\hline Social functioning & $49.6( \pm 7.5)$ & $50.2( \pm 7.5)$ & 0.47 \\
\hline Role emotional & $49.3( \pm 6.2)$ & $49.8( \pm 5.9)$ & 0.66 \\
\hline Mental health & $50.5( \pm 6.0)$ & $50.8( \pm 6.1)$ & 0.99 \\
\hline Physical component summary & $49.4( \pm 6.3)$ & $49.4( \pm 6.8)$ & 0.06
\end{tabular}

EPIC - expanded prostate cancer index composite questionnaire, SF-8 - medical outcomes study 8-item short form health survey questionnaire

A

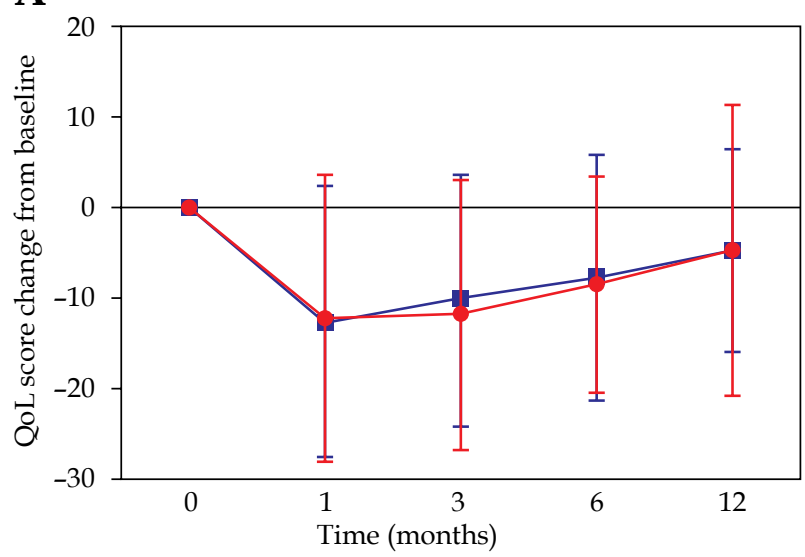

C

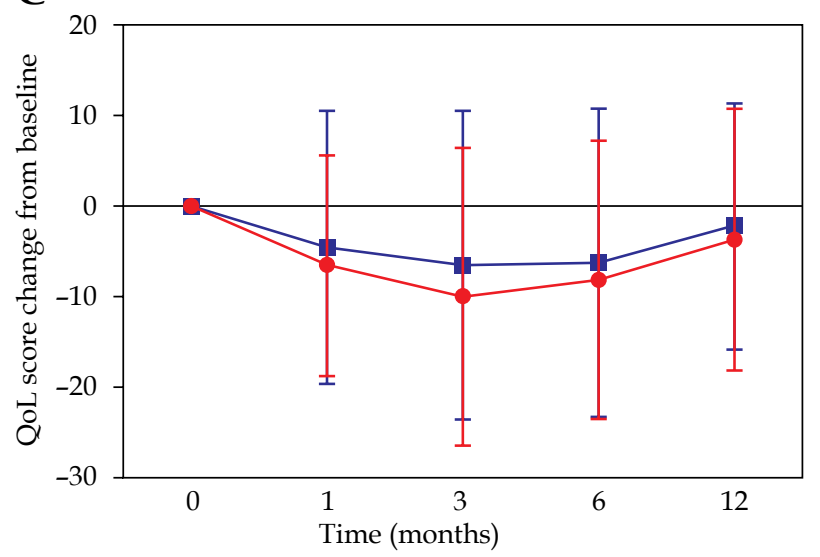

B

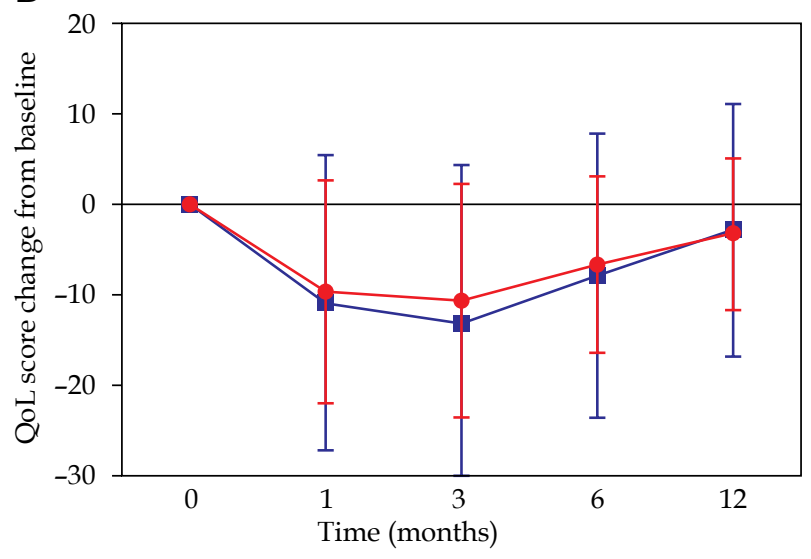

$\mathbf{D}$

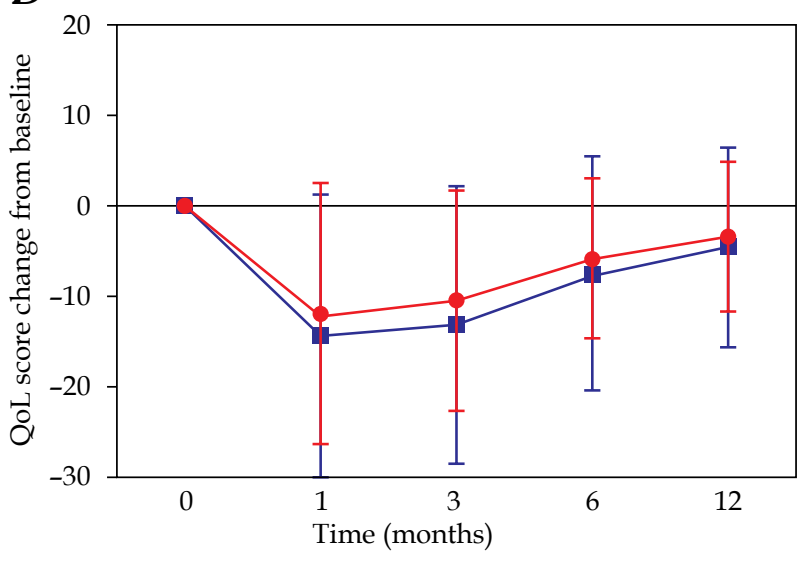

- Celecoxib + Tamsulosin

- Tamsulosin

Fig. 1. Chronological changes in subscales of the urinary domain. A) Urinary function; B) Urinary bother; C) Urinary incontinence; D) Urinary irritative 
A

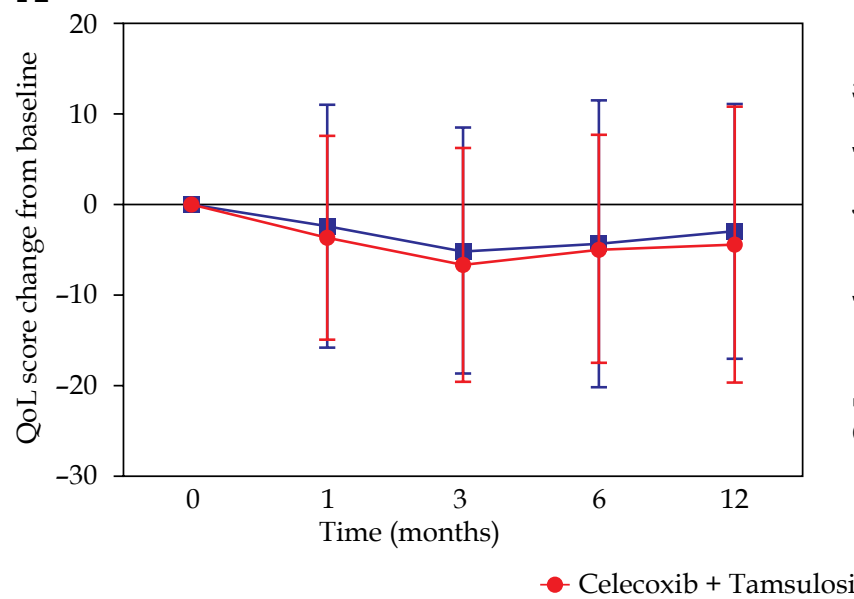

B

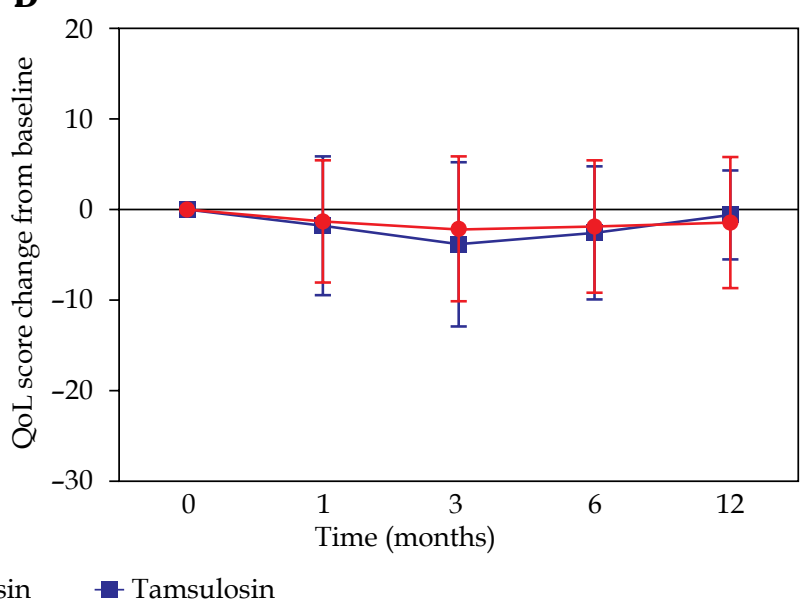

Fig. 2. Chronological changes in subscales of the bowel domain. A) Bowel function; B) Bowel bother

and monotherapy groups were 0.46 (SD: \pm 7.8 ) and 2.03 (SD: \pm 7.7 ), respectively, without a significant difference $(p=0.08)$. There was no significant difference between the mean change in scores from baseline to 6 months $(p=0.49$, combination: mean 2.1, SD: \pm 10.1 ; monotherapy: mean 2.3, SD: \pm 8.5 ) or 12 months ( $p=0.42$, combination: mean 0.24 , SD: \pm 16.4 ; monotherapy: mean 3.3, SD: \pm 8.4 ).

For the sexual domain of EPIC, the mean changes in scores from baseline to 1 month in the combination and monotherapy groups were -2.3 (SD: \pm 7.6 ) and -3.0 (SD: \pm 9.5$)$, respectively, and no significant difference was found between the two groups $(p=0.47)$. The mean changes in scores from baseline to 3 months in the tamsulosin plus celecoxib and tamsulosin groups were -1.7 (SD: \pm 9.8 ) and -2.5 (SD: \pm 11.4$)$, respectively, without a significant difference $(p=0.50)$. There was no significant difference between the mean changes in scores from baseline to 6 months $(p=0.46$, combination: mean -1.5 , SD: \pm 11.4 ; monotherapy: mean -2.0 , SD: \pm 11.2 ) or 12 months $(p=0.66$, combination: mean $-1.8, \mathrm{SD}: \pm 12.5$; monotherapy: mean -1.3 , SD: \pm 11.9$)$.

For the PCS scores of SF-8, the mean changes in scores from baseline to 1 month in the combination and monotherapy groups were 0.009 (SD: \pm 7.2 ) and -0.89 (SD: \pm 7.9 ), respectively, and no significant difference was found between the two groups $(p=0.29)$. The mean changes in scores from baseline to 3 months in the combination and monotherapy groups were -0.32 (SD: \pm 6.8 ) and -0.22 (SD: \pm 7.2 ), respectively, without a significant difference $(p=0.90)$. There was no significant difference between the mean changes in scores from baseline to 6 months $(p=0.42$, combination: mean -0.29 , SD: \pm 8.4 ; monotherapy: mean -0.44 , SD: \pm 8.8 ) or 12 months $(p=0.16$, combination: mean -0.21 , SD: \pm 9.8 ; monotherapy: mean -0.55 , SD: \pm 9.3 ) (Figure 3G). For the bodily pain domain (Figure $3 \mathrm{C}$ ), the mean changes in bodily pain from baseline to 1 month in the tamsulosin plus celecoxib and tamsulosin groups were -0.63 (SD: \pm 9.6 ) and 0.13 (SD: \pm 9.8 ), respectively. The mean changes at 3 months in the tamsulosin plus celecoxib and tamsulosin groups were -0.04 (SD: 9.8) and 0.38 (SD: 9.8), respectively.
For the MCS score of SF-8, the mean changes in scores from baseline to 1 month in the combination and monotherapy groups were 0.41 (SD: \pm 7.4 ) and 1.3 (SD: \pm 8.5 ), respectively, and no significant difference was found between the two groups $(p=0.32)$. The mean changes in scores from baseline to 3 months in the combination and monotherapy groups were 0.36 (SD: \pm 6.8 ) and 1.1 (SD: \pm 9.0 ), respectively, without a significant difference $(p=0.44)$. There was no significant difference between the mean changes in scores from baseline to 6 months $(p=0.41$, combination: mean $0.77, \mathrm{SD}: \pm 8.5$; monotherapy: mean 1.3 , SD: \pm 9.5 ) or 12 months ( $p=0.27$, combination: mean 0.13 , SD: \pm 10.1 ; monotherapy: mean -1.7, SD: \pm 9.1 ) (Figure 3).

\section{Discussion}

The present randomized controlled trial was powered according to the primary endpoint (improvement of lower urinary tract symptoms [LUTS] in the IPSS), but the additional effect of the COX-2 inhibitor use on improving IPSS was not found [10]. Furthermore, in this study, no additional effect of COX-2 inhibitor use was found at 1, 3, 6 , and 12 months after seed implantation for the urinary domain of EPIC. Crook et al. conducted a randomized trial to determine whether COX-2 inhibitor regimen initiated 1 week before seed implantation might diminish inflammatory response, and reduce edema, retention rates, and symptom severity. The study found that initiation of COX-2 inhibitor regimen 1 week before brachytherapy compared to initiation immediately after the procedure did not reduce 1-month edema, improve IPSS at 1 or 3 months, or reduce the need for catheterization [20]. Previous reports showed that addition of COX-2 inhibitor reduced daytime urinary frequency and post-void residual, which were secondary endpoints in this study [10]. However, QoL with regard to the urinary domain including IPSS, overactive bladder symptom score, and EPIC (urinary summary domain and subscales) did not improve on using COX-2 inhibitor. Furthermore, there was no significant effect of COX-2 inhibitor on urinary QoL in patients treated with LDR brachytherapy or with 
A

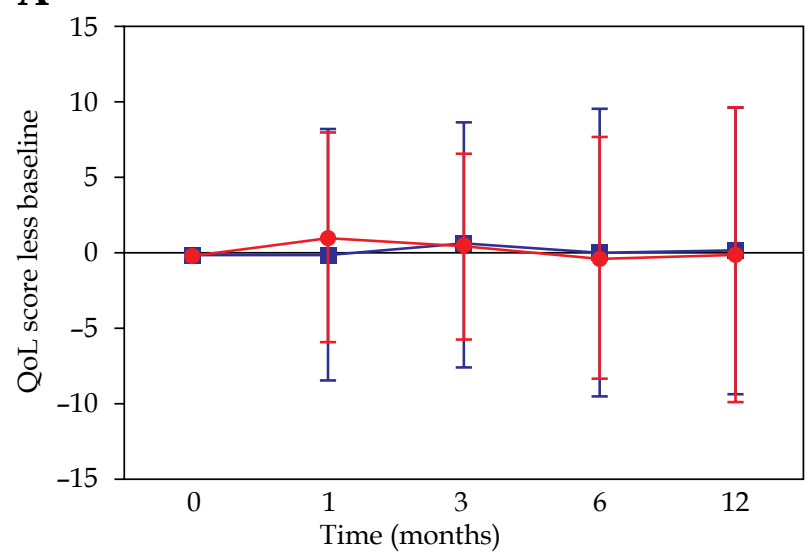

C

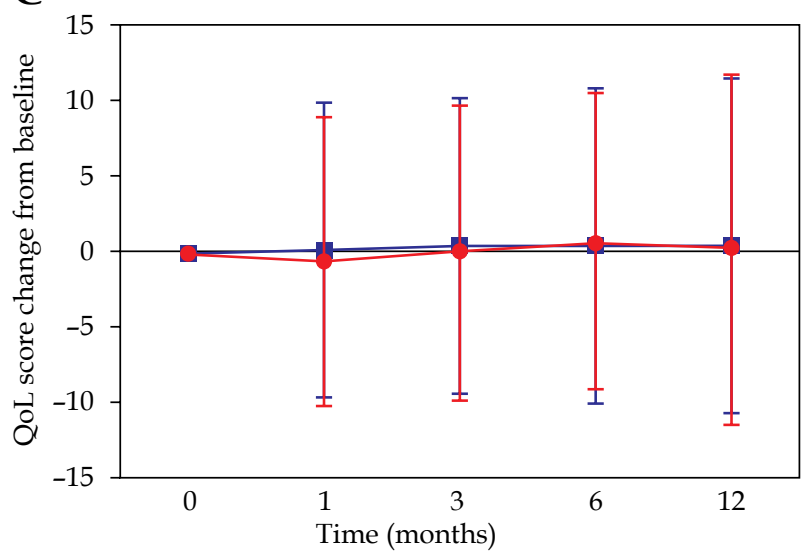

E

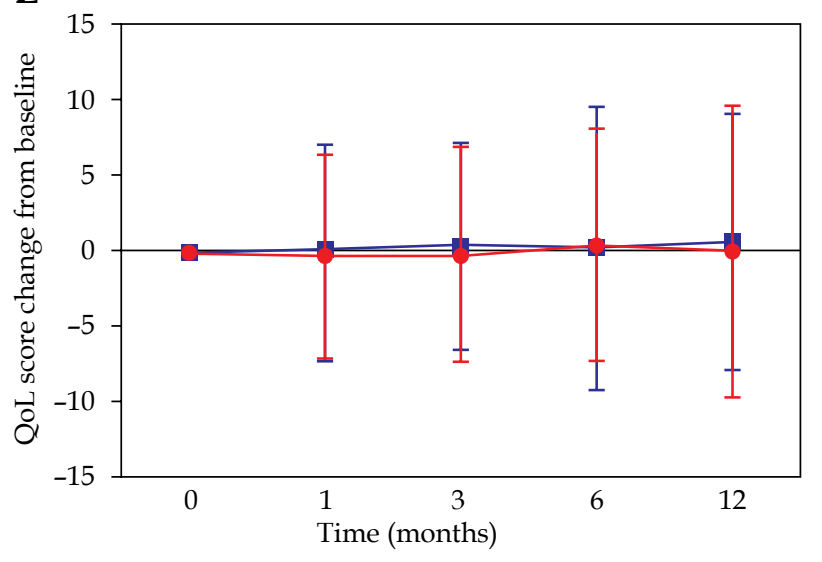

B

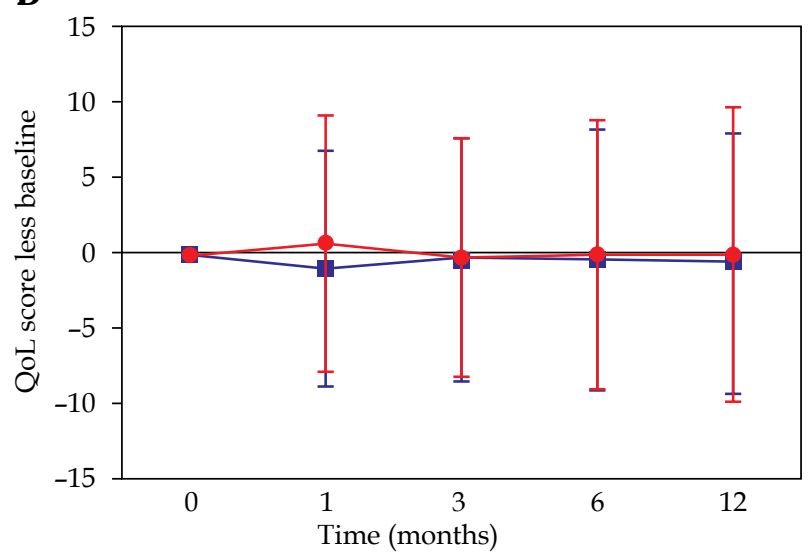

D

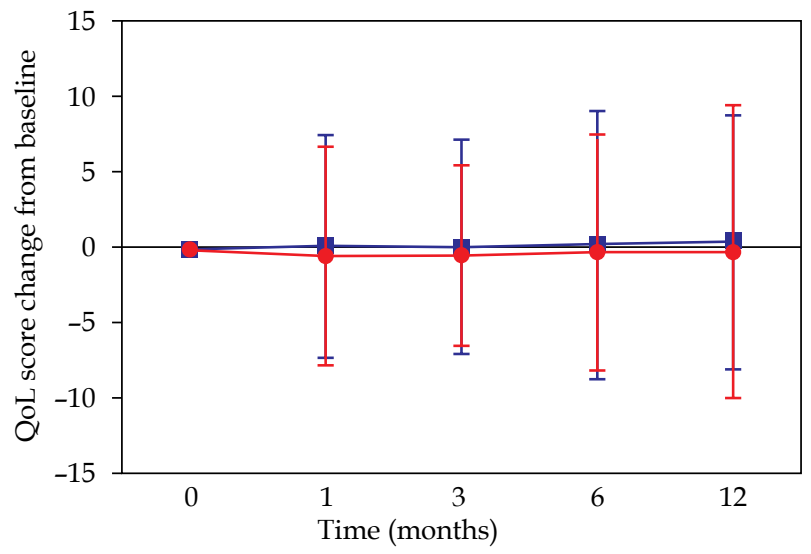

$\mathbf{F}$

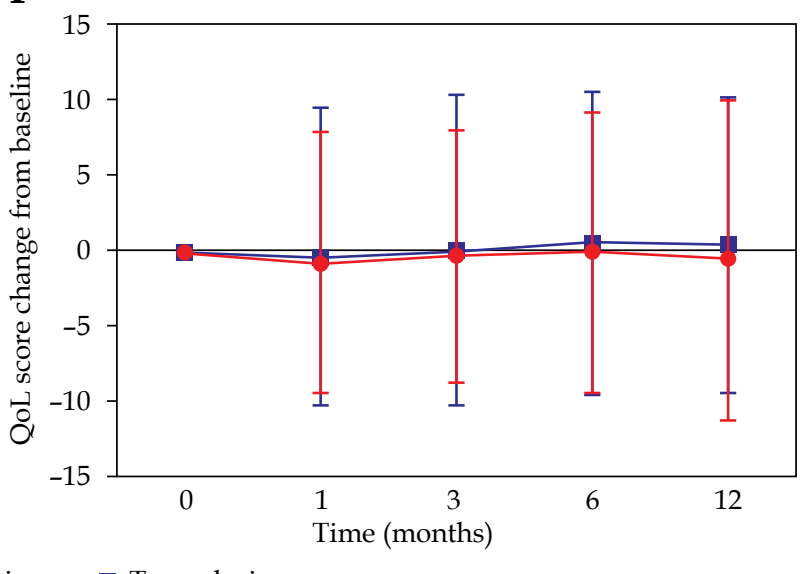

Tamsulosin

Fig. 3. Chronological changes in SF-8 scores. A) Physical function; B) Role physical; C) Bodily pain; D) Vitality; E) General health; F) Social functioning

LDR brachytherapy plus EBRT in the present study (data not shown), although urinary toxicities are different between LDR brachytherapy and LDR brachytherapy plus EBRT $[5,21]$. These results may indicate that the use of COX-2 inhibitor has an effect on urinary symptoms to some extent, but COX-2 inhibitor does not improve QoL in terms of the urination domain. That is, routine use of COX-2 inhibitor for LUTS during the perioperative period of brachytherapy is not warranted.
It is also reported in our other study that QoL for the bowel domain worsened in $37 \%$ patients treated with LDR brachytherapy alone and $68 \%$ patients treated with LDR brachytherapy plus EBRT within 3 months after LDR brachytherapy [5]. Although unlike IMRT, after the use of brachytherapy, QoL for the bowel domain improved after 24 months [5], a worse QoL in terms of the bowel domain is as important as that in terms of the urinary domain. If the lower QoL in terms of the bowel domain is 
G

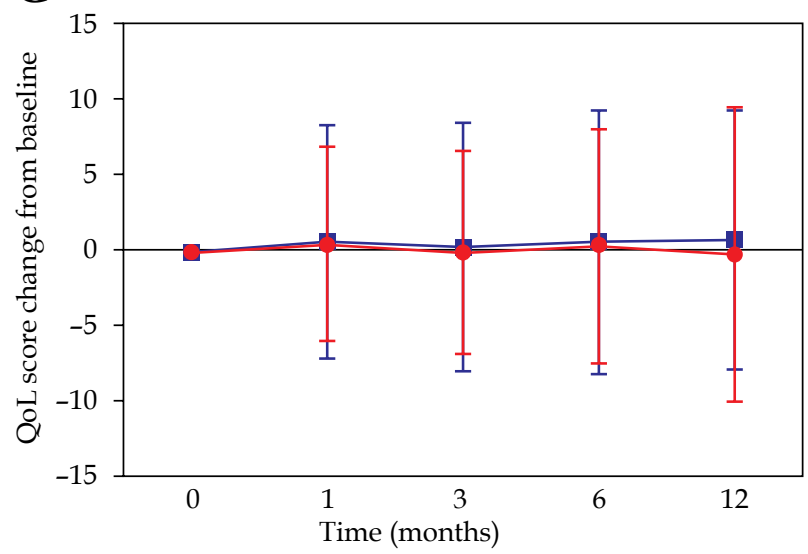

I

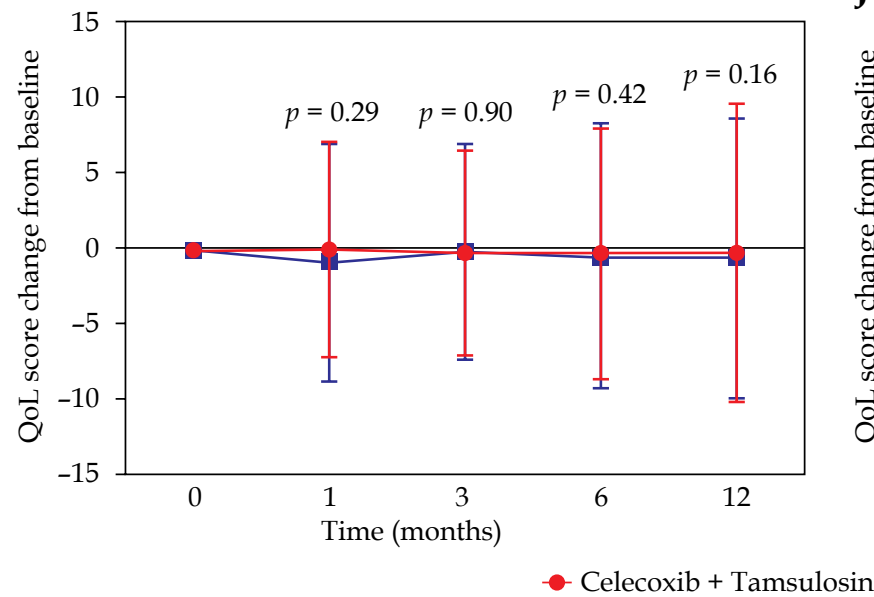

$\mathbf{H}$

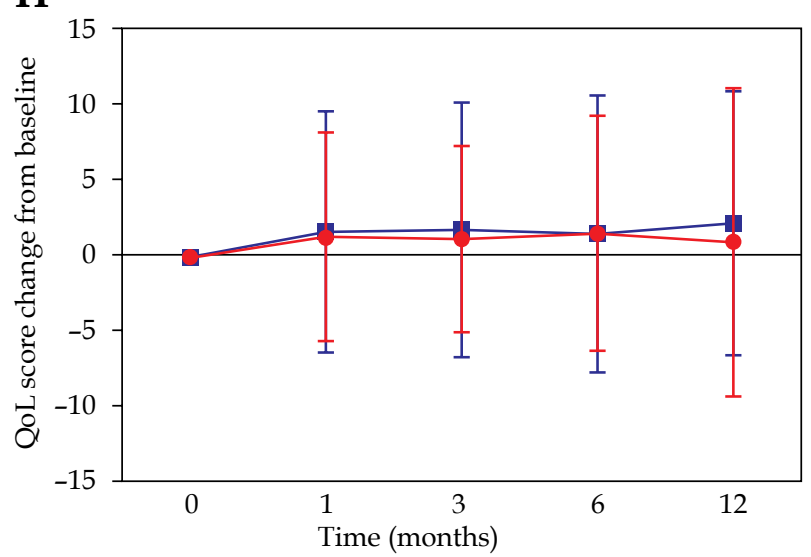

$\mathbf{J}$

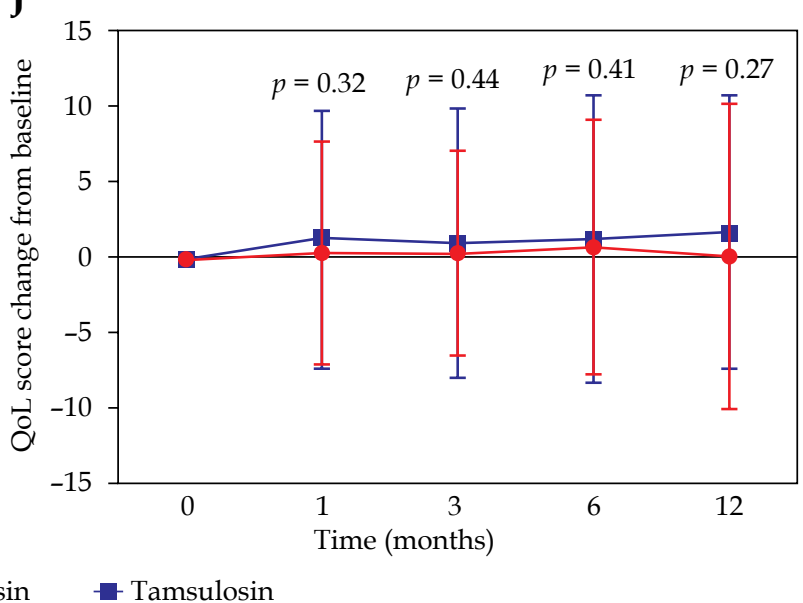

Fig. 3. Cont. G) Role emotional; H) Mental health; I) Physical component summary; J) Mental component summary

caused by inflammation due to a radiation effect, COX-2 inhibitor should be effective in resolving these adverse events. However, the present result showed almost the same changes in both groups (Figure 1B). These results indicate that routine use of COX-2 inhibitor to improve QoL in terms of the bowel domain during the peri-operative period of brachytherapy is not warranted.

Another concern is with regard to the analgesic efficacy of COX-2 inhibitor. In some reports, COX-2 inhibitor was found to relieve pain during peri-operative period; it was also found to improve QoL in a randomized controlled study [22]. Therefore, COX-2 should improve pain and physical and mental QoL. However, there was no significant difference found between physical and mental QoL upon combination therapy with the COX-2 inhibitor (Figure $3 \mathrm{G}$ and $3 \mathrm{H}$ ). Furthermore, even in the bodily pain domain, there was no significant difference found with COX-2 inhibitor use (Figure 3C). These results indicate that routine use of COX-2 inhibitor to reduce pain during peri-operative period of brachytherapy is not warranted.

The present trial did not show efficacy of the COX-2 inhibitor in improving QoL within 12 months after seed implantation, which may be attributed to some reasons. The first reason is the timing of QoL evaluation. Gan et al. and Brattwall et al. reported the efficacy of COX-2 inhib- itor in improving QoL within 7 days after laparoscopic cholecystectomy and elective hallux valgus surgery $[19,23,24]$. Brattwall et al. reported that 16 weeks after laparoscopic cholecystectomy, the efficacy of COX-2 inhibitor disappeared [24]. Given these findings, the COX-2 inhibitor may be effective in improving QoL immediately after surgery (within 7 days after the procedure), although the present trial did not evaluate QoL within 7 days after seed implantation. The second reason is that the dosage of COX-2 inhibitor (200 mg/day) may not be sufficient to improve QoL scores after seed implantation. Fan et al. reported the efficacy of celecoxib ( $400 \mathrm{mg}$ / day) on QoL in patients treated with IMRT for nasopharyngeal carcinoma [13]. The third reason is that the expression of COX-2 in patients treated with LDR brachytherapy may be different from that in patients who were treated with a different variety of radiation therapy or surgery. However, the expression of COX-2 in patients after brachytherapy was not evaluated in the present study and this is one of the limitations of our study.

This study has also other limitations. First, it is an open-label trial and the results of QoL may be affected by the placebo effect. Second, the present trial was powered according to the primary endpoint (IPSS), and the objectives of the present report were the secondary endpoint. 
Finally, compliance and adherence were not evaluated. Adherence was confirmed only verbally at a patient's visit.

\section{Conclusions}

Combination treatment with celecoxib and tamsulosin during peri-operative period is not superior to sole tamsulosin treatment for improving QoL in patients undergoing LDR brachytherapy.

\section{Disclosure}

The authors report no conflict of interest.

\section{References}

1. Grimm P, Billiet I, Bostwick D et al. Comparative analysis of prostate-specific antigen free survival outcomes for patients with low, intermediate and high risk prostate cancer treatment by radical therapy. Results from the Prostate Cancer Results Study Group. BJU Int 2012; 109 Suppl 1: 22-29.

2. Kuban DA, Levy LB, Cheung MR et al. Long-term failure patterns and survival in a randomized dose-escalation trial for prostate cancer. Who dies of disease? Int J Radiat Oncol Biol Phys 2011; 79: 1310-1317.

3. Tanaka N, Asakawa I, Nakai Y et al. Comparison of PSA value at last follow-up of patients who underwent low-dose rate brachytherapy and intensity-modulated radiation therapy for prostate cancer. BMC Cancer 2017; 17: 573.

4. Morris WJ, Tyldesley S, Rodda S, et al. Androgen suppression combined with elective nodal and dose escalated radiation therapy (the ASCENDE-RT Trial): An analysis of survival endpoints for a randomized trial comparing a lowdose-rate brachytherapy boost to a dose-escalated external beam boost for high- and intermediate-risk prostate cancer. Int J Radiat Oncol Biol Phys 2017; 98: 275-285.

5. Nakai Y, Tanaka N, Asakawa I et al. Quality of life in patients who underwent $125 \mathrm{I}$ brachytherapy, ${ }^{125}$ I brachytherapy combined with three-dimensional conformal radiation therapy, or intensity-modulated radiation therapy, for prostate cancer. J Radiat Res 2019; 60: 270-280.

6. Tanaka N, Fujimoto K, Asakawa I et al. Variations in health-related quality of life in Japanese men who underwent iodine-125 permanent brachytherapy for localized prostate cancer. Brachytherapy 2010; 9: 300-306.

7. Merrick GS, Butler WM, Wallner KE et al. Prophylactic versus therapeutic alpha-blockers after permanent prostate brachytherapy. Urology 2002; 60: 650-655.

8. Oyama N, Aoki Y, Ito H et al. Alpha 1-adrenoceptor blocker may improve not only voiding but also storage lower urinary tract symptoms caused by (125) I brachytherapy for prostate cancer. ISRN Urol 2014; 2014: 140654.

9. Tanaka N, Torimoto K, Asakawa I et al. Use of alpha-1 adrenoceptor antagonists in patients who underwent low-doserate brachytherapy for prostate cancer - a randomized controlled trial of silodosin versus naftopidil. Radiat Oncol 2014; 9: 302 .

10. Tanaka N, Torimoto K, Asakawa I et al. Comparison of chronological changes in urinary function in patients who underwent low-dose-rate brachytherapy for prostate cancer -A randomized controlled trial of alpha- 1 adrenoceptor antagonist alone versus combination with cyclooxygenase-2 inhibitor. Brachytherapy 2018; 17: 537-543.

11. Jaal J, Dörr W. Radiation induced inflammatory changes in the mouse bladder: the role of cyclooxygenase-2. J Urol 2006; 175: 1529-1533.
12. Feigenberg SJ, Wolk KL, Yang $\mathrm{CH}$ et al. Celecoxib to decrease urinary retention associated with prostate brachytherapy. Brachytherapy 2003; 2: 103-107.

13. Qiang Fan, Erwen Bao, Jialiang Zhou et al. Celecoxib combined with hippocampus sparing intensity-modulated radiation therapy reduces the injury of cognitive function induced by radiotherapy in patients with nasopharyngeal carcinoma. Int J Clin Exp Med 2017; 10: 12546-12552.

14. Ghasemi A, Danesh B, Yazdani-Charati J et al. Randomized double-blind placebo-controlled trial of celecoxib for the prevention of skin toxicity in patients receiving radiation therapy for breast cancer. Antiinflamm Antiallergy Agents Med Chem 2018; 17: 57-67.

15. Wei JT, Dunn RL, Litwin MS et al. Development and validation of the expanded prostate cancer index composite (EPIC) for comprehensive assessment of health-related quality of life in men with prostate cancer. Urology 2000; 56: 899-905.

16. Ware JE, Kosinski M, Dewey JE, Gandek B. How to score and interpret single-item health status measures: a manual for users of the SF-8 Health Survey. Lincoln RI: QualityMetric Incorporated, 2001.

17. Falahatkar S, Mokhtari G, Pourreza F et al. Celecoxib for treatment of nocturia caused by benign prostatic hyperplasia: a prospective, randomized, double-blind, placebo-controlled study. Urology 2008; 72: 813-816.

18. Davis BJ, Horwitz EM, Lee WR et al. American Brachytherapy Society consensus guidelines for transrectal ultrasound-guided permanent prostate brachytherapy. Brachytherapy 2012; 11: 6-19.

19. Stone NN, Winoker JS, Kaplan SA et al. Factors influencing long-term urinary symptoms after prostate brachytherapy. BJU Int 2018; 122: 831-836.

20. Crook J, Patil N, Wallace K et al. A phase III randomized trial of the timing of meloxicam with iodine- 125 prostate brachytherapy. Int J Radiat Oncol Biol Phys 2010; 77: 496-501.

21. Mukai Y, Hayashi N, Koike I et al. Acute and late toxicities in localized prostate cancer patients treated with low-dose 125I brachytherapy (110 Gy) in combination with external beam radiation therapy versus brachytherapy alone (160 Gy). J Contemp Brachytherapy 2018; 10: 397-404.

22. Gan TJ, Joshi GP, Zhao SZ et al. Presurgical intravenous parecoxib sodium and follow-up oral valdecoxib for pain management after laparoscopic cholecystectomy surgery reduces opioid requirements and opioid-related adverse effects. Acta Anaeshesiol Scand 2004; 48: 1194-1207.

23. Gan TJ, Joshi GP, Viscusi E et al. Preoperative parenteral parecoxib and follow-up oral valdecoxib reduce length of stay and improve quality of patient recovery after laparoscopic cholecystectomy surgery. Anesth Analg 2004; 98: 1665-1673.

24. Brattwall M, Turan I, Jakobsson J et al. Pain management after elective hallux valgus surgery: a prospective randomized double-blind study comparing etoricoxib and tramadol. Anesth Analg 2010; 111: 544-549. 